\title{
Hermenéutica jurídica y teoría económica
}

Héctor Noejovich Ch.

\section{Introducción}

Hace algunos años, un distinguido economista, Donald McCloskey (1983), escribió un artículo sobre la retórica en la economia ("The Rethoric of Economics»), sacando a la luz una discusión sobre la naturaleza de la ciencia económica y el carácter «teórico» derivado de utilizar la formalización matemática.

Ello trae a colación un chiste, muy profundo a mi entender, que metafóricamente expone el problema: ¿Cuál es la diferencia entre un ingeniero, un economista y un abogado?. A la pregunta: ¿cuánto es dos más dos?, el ingeniero responde, sin ninguna duda: es igual a cuatro; el economista responde: es significativamente distinto de cero; el abogado repregunta ¿̨uánto quieres que sea?

Las respuestas del economista y del abogado son análogas en algún sentido: no tienen definición concreta y están sujetas a interpretación. En cómo se exterioriza esta última radica la diferencia observable entre la Economía y el Derecho, ya que esencialmente estamos frente a ciencias sociales o del espíritu, como decía Dilthey, que no pueden funcionar como ciencias de la naturaleza, dado que su objeto es común: el hombre social, visto tanto desde sus aspectos jurídicos, como desde sus aspectos económicos, integrados e indisolubles dentro de la conducta humana.

La relación entre Economía y Derecho viene de antaño. Los primeros economistas provinieron de las canteras de las Facultades de Derecho. Sólo a partir del desarrollo de instrumentos analíticos basados en las matemáticas, primero, y en la estadística, luego, se fue especia- 
lizando la economía como rama distinta. ${ }^{1}$ Esta división llevó a una conducta independiente en ambas especialidades, desvirtuada solamente por algunas corrientes doctrinarias.

Sin perjuicio de que las ideas sobre el enlace de ambas provienen del siglo pasado, se ha puesto en boga, en las últimas décadas, a partir del teorema de Coase ${ }^{2}$, las ideas de North ${ }^{3}$ y especialmente los trabajos de Posner, el denominado "análisis económico de derecho». El Derecho y la Economía resultarían dos caras de una misma moneda. Mientras el primero provee el análisis institucional, la segunda provee los operadores teóricos. El economista supone en su ceteris paribus un marco institucional dado; la realidad, sin embargo, muestra que ese marco institucional se encuentra en movimiento y se retroalimenta de las relaciones económicas. Es decir, existe una interacción entre ambos.

En los EE.UU. se ha desarrollado, en las últimas décadas, una literatura bastante extensa sobre el tema de Law and Economics, con distintas posiciones sobre el particular. A diferencia de los modelos teóricos, se tratan de ejemplos empíricos que versan sobre marcos institucionales determinados. El quid de la cuestión radica en la relación de los mismos con el contexto social; y, desde un punto de vista metodológico, en cómo integrar ambos enfoques.

\section{Algunos paralelismos conceptuales}

\subsection{La noción de sistema}

Ésta constituye el "primer elemento común». Hablamos generalmente de sistemas jurídicos y de sistemas económicos como si estuviesen divorciados del sistema social. Es claro que por razones analíticas, derivadas de los instrumentos utilizados con ese fin, pueden desglosarse los aspectos jurídicos de los aspectos económicos, los cua-

1 Jevons, Marshall, Walras y Pareto son algunos ejemplos. No obstante, en las cátedras sudamericanas y en los puestos públicos relacionados con la Economía, prevalecieron los abogados hasta bien avanzado el siglo XX.

2 Ronald Coase, "The problem of social cost" en: Journal of Law and Economics, 1961.

3 Douglass North, Instituciones, cambio institucional y desempeño económico, 1993. 
les, sin embargo, deben necesariamente integrarse para completar una conclusión válida.
¿Qué es un sistema?
"Un conjunto de estructuras vinculadas entre sí por ciertas reglas (leyes) [.... estructura es un conjunto de objetos vinculados entre sí según ciertas reglas (leyes) [...] objeto es cualquier realidad posible: individuo, concepto, institución y cosa [...] reglas" "[...] principios explicitos de combinación, de planteamiento de relación entre los elementos de un sistema y las normas intencionalmente creadas y aplicadas para organizar la vida social [...]» (Godelier, 1976: 254).

Esta definición, de corte estructuralista, es utilizada por el autor para tipificar el sistema económico como una articulación entre estructuras de producción, distribución y consumo, donde está inmersa la variable institucional. Formado en las canteras del marxismo, Godelier representa un intento estructuralista de revisión de las ideas de Marx.

Este último, como se sabe, supeditó el sistema jurídico (superestructura en términos de Marx) al sistema económico (estructura en su terminología). En cambio para Weber ${ }^{4}$, de inicio, el orden jurídico pertenece a la esfera del deber-ser, en tanto que el orden económico se refiere a los acontecimientos reales; la integración se verifica en el plano social donde ambos interactúan.

Es aquí donde el sistema se unifica, en el acontecer, quedando los instrumentos teóricos como elementos interpretativos de esa realidad $y$ de ese devenir cotidiano. ${ }^{5}$ Es indudable que, tanto en el campo privado, como en el de la gestión pública, las instituciones juegan un rol fundamental.

4 Max Weber, Economía y sociedad, 1983 [1922], p. 256 y ss.

5 Ese instrumental teórico entraría dentro de los «tipos ideales» weberianos, a los cuales la experimentación les daría el «sentido mentado». Recordemos que el experimento, en las ciencias sociales, no formula proposiciones permanentes, sino aquellas que se ajustan al mismo. Así como en el Derecho existen casos similares, pero no exactamente iguales, en la Economía sucede algo semejante: dos situaciones pueden parecerse al extremo de buscar fórmulas comunes, pero la constatación empírica muestra, especialmente en la aplicación de políticas públicas, que los resultados pueden ser diferentes. En este caso, la historia económica funcionaría como una suerte de «jurisprudencia» en la medida que nos muestra las consecuencias de las políticas públicas. 
Siendo el Derecho de carácter esencialmente coactivo, y la Economía de carácter espontáneo, es claro que el primero limita la libertad de los actos económicos. ${ }^{6}$ Esa limitación está directamente relacionada con las denominadas políticas públicas; es decir, el accionar del Estado que incide en el devenir cotidiano de las actividades de los individuos y sus consecuencias en el orden económico.

Así, volviendo a la definición de Godelier -cf. supra- las normas organizan la vida de los individuos y, por consiguiente, enmarcan la actividad de las estructuras económicas que integran el sistema social en su conjunto.

\subsection{La instrumentación de las políticas públicas}

Estas no son solamente prescripciones que se derivan de proposiciones económicas, ${ }^{7}$ sino que, más bien, son representativas del rol del Estado en la economía, que la sociedad acepta. Y este rol se refiere a la constitución y a la regulación de un orden económico determinado de acuerdo a lo propuesto por Walter Eucken en $1956 .{ }^{8}$ Dicho autor se contrapone a la concepción del orden espontáneo, tan cara a la Escuela de Viena, representada por Von Mises y Von Hayek.

Esos principios «constitutivos» del orden económico provienen del desarrollo del Estado como organización que maneja el poder, donde la dimensión política es entendida como la concepción que tiene la sociedad del Estado, en tanto «la forma superior y más poderosa de organizar el poder dentro de la sociedad.»"

Dentro de los sistemas constitucionales actuales, que al menos teóricamente siguen el principio de división de poderes, esa instrumentación se realiza a través de:

6 Como ejemplo mencionemos las actividades consideradas ilegales, como la explotación de la coca y sus derivados. Se trata de una actividad económica espontánea al menos por parte de los campesinos, la cual, sin embargo, es prohibida coactivamente por la sociedad, mediante normas jurídicas.

7 Adolfo Figueroa, Teorías económicas del capitalismo, 1992, p. 30.

8 Cfr. Walter Eucken, «El orden de la competencia y su realización» (1956) en: DURR 1983 , p. 43.

9 Marcial Rubio, El sistema jurídico. Introducción al Derecho, 1988, p. 29 y ss. 


\section{(a) Las leyes y dispositivos emanados del poder legislativo}

Esto supone un conjunto normativo coherente, sustentado en el ordenamiento constitucional, que da forma activa a la política económica. Aquí se produce el primer encuentro "práctico» entre orden jurídico y orden económico, toda vez que la redacción de las normas supone recoger prescripciones normativas económicas surgidas de un contexto metodológico distinto al del derecho, el cual servirá de expresión de aquellas.

(b) Las medidas administrativas provenientes del poder ejecutivo.

Tomemos a éstas como el conjunto de "toma de decisiones", en los distintos niveles (Presidente, Gabinete, Ministros, Directores). En otros términos, se supone la existencia de un plan de política económica, sea regulatorio, sea desregulatorio, cuya ejecución corresponde a esos organismos.

\section{(c) Las operaciones de mercado abierto ${ }^{10}$}

Mientras en los dos primeros casos se modifica el marco institucional, en este tipo de actividad, los organismos públicos intervienen directamente en el quehacer económico propiamente dicho. Estos instrumentos actúan, a su vez, en distintas áreas de política económica y sus conceptos teóricos son provistos por la macroeconomía, la teoría del crecimiento y la teoría del desarrollo.

\subsection{La ejecutoriedad" de las normas y su relación con los costos de transacción}

En el ámbito privado, definido el marco institucional y su dinámica de transformación, la ejecución de las normas es de crucial importancia. Allí interesa la efectividad del sistema de administración de justicia, cuya actuación tiene indudablemente efectos económicos. En el campo del Derecho, importa la interpretación jurídica, la cual puede o

10 Por ejemplo la compraventa de dólares que realiza el BCR para regular su cotización.

11 Utilizo "ejecutoriedad" como traducción de "enforcement", palabra inglesa de contenido mucho más amplio. Podemos traducirla también como la "capacidad o efectividad de ejercer coactivamente el cumplimiento de una norma jurídica». 
no coincidir con la interpretación económica; surge, así, un problema que para mí es esencialmente metodológico.

Se discute sobre el supuesto de perfección o imperfección de la ejecutoriedad. Esto obedece tanto a la eficiencia de los contratos, como a la función de utilidad de los agentes involucrados. La ejecutoriedad de las normas, en tanto capacidad de hacer cumplir los contratos y respetar los derechos, depende de las convenciones de las partes y del sistema institucional. En un sistema cooperativo, aun frente a restricciones informales de tipo cultural -como en las sociedades tribales-, pueden existir soluciones eficientes.

Sin embargo, en la medida que las instituciones evolucionan y las sociedades se hacen más complejas, se hace necesario la intervención de un tercero, con poder coercitivo: en este caso es el Estado. La cuestión pasa, entonces, por la composición de ese Estado, dado que quienes ejerzan la fuerza coercitiva, presionarán para satisfacer sus propios intereses.

Por otra parte, el proceso de transformación no sólo depende de la tecnología, sino de las instituciones, las cuales, de esta manera, juegan un rol significativo en los costos de producción. Al lado del significado de las instituciones en los costos de transformación está la incidencia en los costos de transacción y/o negociación. Este costo se mide por la ejecutoriedad de los contratos, de donde las restricciones informales inciden en el contrato real. Mientras más ambiguas y menos claras sean las reglas, mayores serán esos costos.

Estos costos de negociación están inmersos en los costos del mercado (p.e. honorarios, corredores, etc.). De esta forma existen instituciones que suben los costos e instituciones que los bajan.

En consecuencia, el marco institucional afecta tanto los costos de transformación, como los de transacción. Desde este punto de vista, la empresa concebida por la teoría microeconómica es una mera abstracción; la empresa real difiere, notablemente, en la medida que incluye sus problemas de organización, supervisión, monitoreo y medición. $^{12}$

«El marco institucional real es de hecho una mezcla de instituciones que promueven actividades de elevación de productividad y de institucio- 
nes que establecen barreras a la entrada, que alientan restricciones monopolistas y que impiden el flujo barato de la información"» (íbidem: 89).

En definitiva los costos de transacción nos permiten «medir» el marco institucional, especialmente en los casos referidos a las falencias en el cumplimiento obligatorio de los contratos y al respeto de los derechos de propiedad. Algunos de estos costos son difíciles de "medir" (colas, coimas, etc.) y hacen complicado el cálculo del costo de transacción; por ende, dificultan la apreciación de los costos totales. La conclusión que ofrece North es la siguiente:

«[...] el marco institucional desempeña una función importante en el rendimiento de la economía $[\ldots])^{13}$

\section{Confrontaciones metodológicas}

\subsection{Las teorías "puras»}

Figueroa (1992: 32) califica de "análisis puro" al que utiliza teorías económicas que suponen no modificar el marco institucional, y «análisis dialéctico", al que admite la modificación ${ }^{14}$. Entre sus conceptos metodológicos (ibidem), resalta la diferenciación entre proposiciones a, b, g. Las primeras, axiomáticamente establecidas en el "análisis puro» (incluyen marco institucional estable y racionalidad económica ${ }^{15}$ ); las segundas, derivadas lógicamente de las primeras. Las proposiciones $\mathbf{g}$ serían las «normas de política económica», deducidas en ese razonamiento proposicional

Pero en el Derecho sucede algo semejante:

«La teoría pura del derecho es una teoría [...] del derecho en general [...]. Es una teoría general del derecho y no una interpretación de tal o

\section{Ibidem.}

14 Diferencia que aplica a la teoría clásica, cuyo exponente es el materialismo dialéctico, de la teoría neoclásica, es decir la posterior a Marx. En ese sentido pienso que el neoinstucionalismo también es una suerte de análisis que involucra la dinámica del marco institucional sin entrar en el análisis del materialismo dialéctico (cf. Noejovich, 1996). 
cuál orden jurídico [...]. Es una ciencia del derecho y no una política jurídica $[. ..] .{ }^{16}$

En ambos casos, parece claro que el intento es despercudirse de valoraciones subjetivas y ponerse en una suerte de "meta-derecho" y "meta-economía». Tanto en el razonamiento "puro" económico, como en el jurídico, funciona el silogismo hipotético $P \rightarrow Q$. En Economía las $P$ serán las proposiciones a que indica Figueroa. Estas coinciden con las $P \rightarrow Q$ del Derecho, toda vez que representan al marco institucional.

Es decir que, el conjunto $\{P \rightarrow Q\}$ del ámbito del Derecho, sería igual al conjunto de $\{$ a $\}$ proposiciones antes mencionadas; es decir que ambos conjuntos representan al «marco institucional». Consecuentemente, el «análisis dialéctico» señalado para el aspecto económico cf. supra-, es trasladado hacia la confrontación, entre la interpretación del marco institucional, correspondiente al Derecho, y la interpretación que el economista realiza de los hechos económicos.

La interpretación que realiza el Derecho, a través de la administración de justicia (incluyendo la jurisprudencia, la costumbre y la doctrina), tiene efectos sobre el marco institucional, siendo la teoría "pura» el esquema de razonamiento conceptual. En cambio, la que realiza el economista, sobre los hechos económicos en términos de la denominada "economía positiva», no tiene efectos en el marco institucional; sólo tendrán efectos distintos aquéllas que, a través de juicios de valor económico, induzcan a modificaciones institucionales conducentes a determinadas políticas económicas. ${ }^{17}$

\subsection{Causalidad, imputación e interacción}

La causalidad es frecuentemente confundida con el mero «nexo lógico». Así, el $P \rightarrow Q$, es tomado como «nexo causal». Esa confusión lleva al determinismo que establece relaciones "constantes y unívocas», que

15 En este punto se debe tener en cuenta la diferenciación clara de Godelier entre la "racionalidad del sistema» y la "racionalidad de los agentes" (1976:11).

16 Hans Kelsen, Teoría pura del Derecho. 1965, p. 15.

17 Cfr. Martín Bronfenbrenner, «Introducción a la metodología económica para lectores de pretensiones intelectuales medias" en: KRUPP, 1973. 
resultan unilaterales y asimétricas, respecto de la «complejidad organizada". La causalidad, en el campo de las ciencias sociales, es una forma particular de la interacción de los fenómenos (cf. Vázquez-Presedo, 1984: 57 y ss.).

En la denominada economía positiva, el silogismo hipotético es sometido a los consabidos «test de hipótesis», suerte de juzgamiento o interpretación de la "complejidad organizada". Si el test rechaza la formulación $P \rightarrow Q$, resulta cierta $P \rightarrow \sim Q$.

Kelsen (1965: 16 y ss) diferencia entre la causalidad y la imputación en el terreno jurídico, reservando la primera categoría para las ciencias de la naturaleza. En este caso, el $\mathrm{P} \rightarrow \mathrm{Q}$ que representa la norma jurídica, existe una doble conducta humana: la del acto ilícito y la de la sanción que se vincula por la imputación. (ibídem: 20).

Para Rubio (1988: 77 y ss), la norma jurídica, en la formulación $P \rightarrow Q$, expresada como nexo lógico-jurídico entre supuesto y consecuencia, deriva en tres posibles relaciones, según se verifiquen en la realidad cada uno de los elementos del silogismo enunciado. Puede no darse el supuesto $y$, por tanto, no se verifica la consecuencia, pero el silogismo derivado de la norma jurídica sigue siendo válido.

Dado el supuesto debe verificarse la consecuencia, pero ello es una necesidad lógica que puede no realizarse ${ }^{18}$. Finalmente, la posibilidad restante es que se verifiquen tanto el supuesto, como la consecuencia (ibidem).

Algo semejante podemos plantear en Economía; la denominada «ley de la demanda", ¿qué nos dice?: "dado los gustos, preferencias e ingresos de los consumidores y que los precios relativos de los demás bienes permanezcan constantes, la demanda de un bien es una función inversa del precio del mismo".

Estamos ante un silogismo hipotético semejante al jurídico, porque aun la consecuencia enunciada conoce de excepciones ${ }^{19}$. Claro está que, la formulación anterior, es considerada de equilibrio parcial y, por consecuencia el análisis económico debe recurrir al equilibrio general, donde se interrelacionan los precios de todos los bienes. Igual sucede con las normas jurídicas, cuya interpretación se realiza en el contexto del ordenamiento global.

18 Como el caso de un criminal que no es atrapado y por ende juzgado conforme a la norma jurídica establecida.

19 El caso Giffen o de los bienes inferiores. 
En ambos casos, la interacción de la "complejidad organizada", involucra la formulación de "nexos causales». La experimentación, cuantitativa en la Economía, jurisprudencial en el Derecho, no es sino una forma de aprehensión de la realidad, la misma que es cambiante. Esto no es sino el "sentido mentado" weberiano del experimento para las ciencias sociales.

Finalizando esta sección veamos un ejemplo en nuestro país de interacción:

"La libre competencia implica que los precios de la economía resultan de la oferta y la demanda, de acuerdo con lo dispuesto por la Constitución y las leyes (Decreto Legislativo $\mathrm{N}^{\circ} 757$, Artículo $4^{\circ}$ )"

Para comenzar, en términos de "análisis económico puro", la fijación de precios por la oferta y demanda no implica la libre competencia. En mercados oligopólicos, por ejemplo, también se forman los precios por la oferta y la demanda; y sin embargo estos mercados son la negación de la libre competencia.

De otro lado, el énfasis normativo «de acuerdo con lo dispuesto» es inverificable, toda vez que es inconsistente con la formulación económica que pretende imbricar. ¿Es esto un «análisis dialéctico» como indica Figueroa (1992: 32)? Por supuesto que no. Simplemente el $P \rightarrow Q$ jurídico es incongruente con el $\mathrm{P} \rightarrow \mathrm{Q}$ económico; en este caso no podemos tomar el marco institucional, en tanto referido a la norma jurídica señalada, como proposición a dentro del ceteris paribus del economista ${ }^{20}$.

\subsection{La interpretación y la teoría}

Según Rubio (1988: 224):

"La teoría de la interpretación jurídica es un conjunto (a veces no muy armónico) de proposiciones de cómo aclarar el sentido de las normas jurídicas $[\ldots] . »$

20 Ello deriva con la aplicación efectiva de la norma, en las discusiones y conflictos sustentados ante INDECOPI. ¿Cómo categorizar la libre competencia? ¿Bajo supuestos económicos? ¿Bajo supuestos jurídicos? 
Podemos contrastarla con la afirmación de Bronfenbrenner (1973: 6):

"Viner: "economía es lo que hacen los economistas", o bien la de mi hija, para quién "economía es donde papá dibuja esas líneas y escribe esos números"».

Por otra parte, para MacCloskey (1985: 85):

«Las pruebas de la ley de la demanda son fundamentalmente literarias. La economía es, pues, científica, pero también literaria».

Asumida la «literalidad» de la economía y la significación de la argumentación interpretativa, podemos trazar un paralelo entre los métodos de interpretación. Pasando revista a los de interpretación jurídica -literal, lógico, sistemático comparativo y por ubicación, histórico y sociológico- (Rubio 1988: 243/260) encontramos que también tienen cabida en la Economía.

Cuando se debaten las teorías monetarias sobre la inflación, por ejemplo, se recurre invariablemente a los ejemplos de otros países y a las soluciones de otros países; pero, sin perjuicio de ello se invoca también una "norma económica", como por ejemplo la "teoría cuantitativa del dinero» o la «teoría keynesiana de la preferencia por la liquidez", las cuales son tomadas como proposiciones teóricas. Los economistas realizan, a través de sus modelos y comprobaciones empíricas, una «interpretación de esas proposiciones económicas".

En definitiva, las conclusiones expresadas en materia de política económica, se asemejan a un «fallo» cuya discusión pertenece al «mercado de los votos o mercado político". Sustancialmente no hay mayor diferencia en la estructura metodológica, sino en la formulación de la argumentación. Parecería que el argumento numérico es más sólido que el literario, lo cual es falso. El número es un signo cuyo contenido es dado por el contexto en el cual se formula.

$Y$ así como el Derecho tiene sus lagunas jurídicas que llenar, la Economía tiene problemas de datos cuantitativos que resolver. La regla de la analogía es común a ambas disciplinas. De otro lado, no hay nada más parecido a la integración jurídica que la interpolación en una serie de datos incompletos. Así como existen vacíos legales, también existen vacíos de información cuantitativa. 
Siguiendo a Olivera, (1997: 4), la Economía es:

"La ciencia que trata de la actividad económica, la ciencia económica, es por consiguiente una ciencia de interpretación, una hermenéutica, y en virtud de la naturaleza de su objeto, una hermenéutica social»(cursiva mía).

Desde este ángulo podemos también unir nuevamente al Derecho y a la Economía dentro de esa hermenéutica social. La dicotomía aparente entre ambas, resulta de una simple taxonomía para efectos analíticos particulares; en realidad como ya señaláramos, constituyen dos caras de una misma moneda.

\section{Reflexiones finales}

La ligazón entre ambas disciplinas evoca indefectiblemente a la Historia. Esa «historia» es, para el Derecho, la jurisprudencia. Los economistas, generalmente, son renuentes a aceptar esos avances, porque prefieren las elucubraciones apriorísticas (cf. Rollinat, 1997). No se trata simplemente de hacer análisis económico del derecho y discutir entre Posner y Calabresi, sino de regresar a las fuentes e incluir las variables institucionales en el quehacer del economista.

Esto es un real desafío, como toda actividad pluridisciplinaria, en la cual todo el mundo reclama la integración, pero nadie quiere abandonar su propia epistemología. Pero en este extremo para mí la cuestión es absolutamente clara y vuelvo a repetirlo: Economía y Derecho son dos caras de la misma moneda.

\section{Referencias bibliográficas}

Bronfenbrenner, Martín.- «Introducción a la metodología económica para lectores de pretensiones intelectuales medias». En: KRUPP (Ed.), 1973.

Coase, Ronald.- "The Problem of Social Cost» en: Journal of Law and Economics, 1, 1961. 
Durr, Ernst, Erhard, Ludwig et. al.- La economía social de mercado: un proyecto económico alternativo. Centro Interdisciplinario de Estudios para el Desarrollo Económico, Buenos Aires, 1983.

Eucken, Walter.- «El orden de la competencia y su realización» (1956). En: Durr, Erhard et. al., 1983.

Figueroa, Adolfo.- Teorías económicas del Capitalismo. Fondo Editorial PUC, Lima, 1992.

Godelier, Maurice.- Racionalidad e irracionalidad en Economía. Siglo XXI, México, 1976.

Kelsen, Hans.- Teoría pura del derecho. Introducción a la ciencia del derecho. EUDEBA, Buenos Aires, 1965.

Krupp, Sherman Roy (Ed.).- La estructura de la ciencia económica. Ed. Aguilar, Madrid, 1973.

Mac Closkey, Donald.- "The Rethoric of Economics», en: Journal of Economic Literature, XXI, 1983.

……. The Rethoric of Economics, 1985.

Noejovich, Héctor Omar.- «Historia económica e institucionalismo: lecciones del historicismo alemán», en: Economía, XIX : 37-38, PUC, Lima, 1996.

North, Douglass.- Instituciones, cambio institucional y desempeño económico. FCE, México, 1993.

Olivera, Julio H. G..- «Realidad e idealidad en la ciencia económica» en: Ciclos, VII: 13, Buenos Aires, 1997.

Posner, Richard A..- Economic Analysis of Law. Little Brown, 1986.

Roemer, Andrés.- Introducción al análisis económico del derecho. FCE-ITAM-SMGE, México, 1994. 
Rollinat, Robert.- «La historia económica y el lugar de las instituciones según D. C. North» en: Ciclos, VII: 13, Buenos Aires, 1997.

Rubio, Marcial.- El sistema jurídico. Introducción al Derecho. Fondo Editorial PUC, Lima, 1998.

Vazquez-Presedo, Vicente.- Economía, ciencia e ideología. Editorial Tesis, Buenos Aires, 1984.

Weber, Max.- Economía y sociedad. FCE, México, 1983 [1922]. 